\title{
Book review: Kritische Landforschung. Konzeptionelle Zugänge, empirische Problemlagen und politische Perspektiven
}

\author{
Franziska Kusche \\ Department of Agriculture Economics, Research Group BioMaterialities, \\ Humboldt-Universität zu Berlin, Berlin, Germany \\ Correspondence: Franziska Kusche (franziska.kusche@hu-berlin.de)
}

Published: 1 February 2021

\begin{abstract}
Maschke, L., Mießner, M., und Naumann, M.: Kritische Landforschung. Konzeptionelle Zugänge, empirische Problemlagen und politische Perspektiven, transcript, Bielefeld, 148ff., ISBN 978-3-8376-5487-5, EUR 19,50, 2021.

Die Corona-Pandemie hat dazu geführt, dass das Leben auf dem Land wieder einmal einen kurzen und oberflächlichen Aufmerksamkeitsschub bekam. Die Sehnsucht nach dem grünen Wohntraum und die Angst vor den Orten der AntiModerne sind die zwei zentralen, wenngleich die einfachsten Erzählstränge, welche die mediale Debatte um das Landleben zu bieten hat.
\end{abstract}

In diese Debatte wagen Lisa Maschke, Michael Mießner und Matthias Naumann eine in jeder Hinsicht notwendige Intervention, welche zugleich den Auftakt einer neuen - gleichnamigen - Reihe zur Kritischen Landforschung des Transcript-Verlages bildet.

Nach der Veröffentlichung des Sammelbandes „Kritische Geographien ländlicher Entwicklung" mit internationalem Fokus konzentrieren sich Naumann und Mießner gemeinsam mit Lisa Maschke in ihrer neuen Publikation auf konzeptionelle Zugänge, empirische Problemlagen und politische Perspektiven ländlicher Transformationen.

Anett Steinführer macht es im Vorwort bereits klar: Eine kritische Forschung und komplexe politische Auseinandersetzung zum ländlichen Raum sind in Deutschland bisher kaum vorhanden. Das Buch beschäftigt sich deshalb mit Fragen der Entwicklungen ländlicher Ökonomien, des sozialen Wandels, Mensch-Umwelt-Beziehungen und Machtverhältnissen.

Anhand einer Revision der englischsprachigen Rural Studies und deren Publikationen möchten die Autor*innen zum einen aufzeigen, dass die dort bereits seit Jahrzehnten statt- findenden Debatten ein wesentlich komplexeres und vielschichtiges Bild der gesellschaftlichen Verhältnisse im ländlichen Raum zeichnen, als das bisweilen die hiesigen Perspektiven tun. Zum anderen sollen Anschlusspunkte gefunden werden, inwiefern diese Arbeiten auch zur „Erklärung und Deutung von Transformationen im ländlichen Raum in der Bundesrepublik helfen können“.

Maschke, Mießner und Naumann stecken ihre Ansprüche für diese Veröffentlichung besonders hoch: eine praktische kritische Gesellschaftstheorie des ländlichen Raumes, die nicht nur für eine breite Leser*innenschaft von Interessierten über Politiker*innen bis hin zu Forschenden angedacht ist, sondern sie wollen Türen zu konkreten Utopien und Alternativen ländlicher Politik und Ländlichkeit öffnen.

Der Aufbau des Buches schließt an die Problemaufrisse und Konzeptionen von „Kritische Geographien ländlicher Entwicklung" an und ist entlang der drei großen Themenfelder wissenschaftliche Konzepte, Themen einer kritischen Landforschung sowie Ansätze alternativer ländlicher Organisation strukturiert.

Gleich zu Beginn werden die Autor*innen programmatisch: Die Probleme, welchen sich ländliche Regionen gegenübersehen, sind ein europaweites Problem. Diese vermeintliche Abkopplung ländlicher Räume bleibt aber nicht länger unwidersprochen und der Niedergang des Ländlichen ist nicht unausweichlich. Das Buch verortet sich in der Tradition einer kritischen (Human-)Geografie, die sich in den vergangenen zehn Jahren einen festen Platz in der deutschsprachigen Academia sichern konnte. Beiträge, die bisher in einschlägigen Reihen erschienen, befassen sich allerdings fast ausschließlich mit Städten, während die Transformationen im ländlichen Raum einer undifferenzierten Debatte zwi- 
schen verklärter grüner Idylle und Restraum der Moderne überlassen wurden. Die Konsequenzen dieser Debatte zeigen sich laut Analyse der Autor*innen auch in dem Erstarken populistischer und reaktionärer Strömungen fernab der urbanen Räume. Die Veränderungen, denen Land und Dorf ausgesetzt sind, sollten deshalb wissenschaftlich begleitet werden, um alternative Handlungsspielräume aufzuzeigen. Die Literaturstudie setzt dazu an den strukturellen Problemen und an den politischen Auseinandersetzungen ländlicher Räume an - vor allem, aber nicht ausschließlich im Globalen Norden.

Was aber sind ländliche Räume? Die Autor*innen sagen sich kurzerhand von den üblichen Indikatoren der Raumplanung los und plädieren im Sinne einer kritischen Gesellschaftstheorie für eine offene Definition ländlicher Räume. Das Ländliche sei nicht per se vorhanden, sondern es wird durch gesellschaftliche Praktiken und Diskurse dazu gemacht. Forschungspraktische und politische Implikationen dieser Definition werden hier aber vorerst nicht besprochen.

Das erste Kapitel widmet sich dahingehend ausschließlich den konzeptionellen und theoretischen Zugängen, welche eine Basis für eine kritische Landforschung bilden können. Auch hier wird an die bisherigen Überlegungen der vorangegangenen Publikation angeschlossen, und es werden Ansätze der Politischen Ökonomie und Ökologie sowie Diskurse und Wahrnehmung ländlicher Räume diskutiert.

Mit einem kurzen Einstieg zur Agrarfrage bei Marx skizzieren die Autor*innen die Anfänge einer modernisierungstheoretischen Sichtweise zum ländlichen Raum, der aufgrund der Industrialisierungstendenzen als Raumkategorie nur als ein vorübergehendes Phänomen in den Homogenisierungsbestrebungen des Kapitalismus wahrgenommen wurde. Eine Vereinheitlichung allen Raumes hin zum Industrialisierten und Urbanen ist aber nicht zu beobachten, und auch das Ländliche in sich sei sehr unterschiedlich, dynamisch und differenziert. Stadt und Land werden als funktionale Raumkategorien von der kapitalistischen Produktion strukturiert, wenn auch auf unterschiedliche Weise. Dies konzeptionieren die Autor*innen unter den Aspekten der ungleichen Entwicklung, der Akkumulation durch Enteignung, der Nahrungsregime und der Umweltgerechtigkeit. Es ermöglicht, den ländlichen Raum als funktional für Inwertsetzungs-, Akkumulations- und Entwertungszyklen zu verstehen. Nicht nur in Form von Klassen- und Einkommensunterschieden, sondern auch in Bezug auf räumliche und ökologische Ungleichheiten, die Folge und Voraussetzung kapitalistischer Entwicklung sind.

Das zweite Kapitel "Transformationen" kontextualisiert und verallgemeinert auf 63 Seiten die Komplexitäten ländlicher Ökonomien, des sozialen Wandels, der MenschUmweltbeziehungen und der Machtverhältnisse und nimmt mit einem empiriegeleiteten Fokus vollkommen zurecht die Hälfte des Buches ein.

Unter ländliche Ökonomien erfassen die Autor*innen die Phänomene des Strukturwandels. Sehr detailliert wird von der Industrialisierung, Internationalisierung und Finanziali- sierung der Landwirtschaft über den Ressourcenextraktivismus gezeigt, dass nicht nur Arbeitsverhältnisse und -weisen im Wandel begriffen sind, sondern auch die soziale Zusammensetzung, und Alltagspraktiken dem Druck der Kommodifizierung ländlicher Räume unterworfen werden. Dies führt nicht nur zur Verschärfung ungleicher Einkommens-, Besitzund Vermarktungsverhältnisse, sondern es verkoppelt auch ländliche Räume im Globalen Norden und Süden über asymmetrische (politische) Handelsregime und setzt sie in eine Konkurrenz der ,günstigeren“ Standortvorteile.

Anhand einer Vielfalt von Themen unter der Kategorie des sozialen Wandels werden die Komplexität der gesellschaftlichen Verhältnisse sowie blinde Flecken der Sozialpolitik aufgezeigt und dabei einige unerwartete Studienergebnisse präsentiert.

Armut und Wohnungsfrage sind zunehmend Probleme, welche auch den ländlichen Raum betreffen, und auf die Veränderung der ökonomischen und Sozialstruktur zurückzuführen sind. Leerstand und ländliche Gentrifizierung, soziale Ungleichheitsverhältnisse, die sich in Rassismus und patriarchalen Strukturen widerspiegeln, Mobilität und Migration - all diese Aspekte zeigen, dass die Ideen von der nachholenden Entwicklung des ländlichen Raumes unter den gegebenen Verhältnissen vor allem eine Mär zugunsten weniger bleibt, die von der Verlagerung und Externalisierung von Ungleichheitsverhältnissen zwischen Stadt und Land profitieren.

Entgegen der Vorstellungen der grünen Idylle kann der Abschnitt Mensch-Umwelt-Beziehungen aufzeigen, wie gerade der Anspruch an die Funktionalität ländlicher Räume als Rohstofflieferant und ökologischer Schutzraum mit Widersprüchen und Spannungen einhergeht. So leiden ländliche Regionen unter Umständen unter wesentlich erhöhten Umweltbelastungen und Zerstörung aus der Landwirtschaft, dem Bergbau oder Industrieanlagen - und sind durch diese Ökonomien abhängiger und anfälliger für die Veränderungen des Klimawandels.

Die Machtverhältnisse, welche all diese Aspekte durchziehen, werden unter drei Aspekten kategorisiert: die der räumlichen Maßstabsebenen und Akteure, der verfolgten Entwicklungsstrategien und der Bedeutung populistischer Bewegungen. Vor allem, dass die Paradigmen ländlicher Entwicklung - extern geleitete Modernisierung vs. endogene Entwicklung - erst zu Ende des Buches aufgegriffen werden, verwundert hier ein wenig. Trotzdem ist gerade die kritische Betrachtung der endogenen Entwicklungsansätze differenziert dargestellt.

Die wachsende Zustimmung autoritärer Bewegungen und Parteien erhält dann zum Ende dieses Kapitels nochmals anhand des US-amerikanischen und einzelner europäischer Beispiele eine differenzierte Betrachtung, die man sich in der deutschen Debatte wünschen würde. Ohne die Hinwendung zu menschenverachtenden politischen Positionen zu rechtfertigen, zeigen die Autor*innen mit den ausgewählten Studien, wie die Transformationen des ländlichen Raums, gesell- 
schaftliche Diskurse sowie eine linke Ignoranz dazu beitragen, das ländliche Spielfeld rechten Parteien zu überlassen.

Der abschließende Teil des Buches will sich Alternativen und Utopien widmen und zeigen, dass auch ländliche Räume Orte von emanzipatorischen Widerständen sind. Konzeptionell fokussieren sich die Autor*innen auf die Themen Selbstorganisation und Gemeinschaftsgüter sowie deren Akteur*innen. Erstere wird dargestellt in ihrer Ambivalenz zwischen emanzipatorischer und selbstbestimmter Krisenbewältigung verdünnter Daseinsversorgung und neoliberaler Selbstausbeutung ehrenamtlicher Arbeit, die gerade im ländlichen Raum immer mehr und zwanghaft institutionalisiert wird. Ein besonders interessanter Aspekt - die territoriale Selbstkontrolle - wird nur kurz angerissen. Das eigene Territorium zu kontrollieren, ermöglicht es Ländlichkeit in einem eigenen, antikapitalistischen oder eben auch reaktionären Sinne zu definieren. Territorialität ist in spanischsprachigen, vor allem lateinamerikanischen Kontexten ein zentrales Konzept und Thema von Selbstbestimmung und Organisation ländlicher Räume und Gemeinschaften. Hier öffnen sich sehr weite Anschlussstellen für linke emanzipatorische Politiken, auch wenn territoriale Selbstbestimmung im deutschen Kontext momentan schwer durch die Reichsbürgerund Anastasia-Bewegung belastet ist. Umso wichtiger ist es, progressive Alternativen zu präsentieren.
Hier wären statt einer ausschweifenden Darstellung von Bookchins libertärem Munizipalismus und konzeptionellen Debatten zu Ostrom eher verstärkte empirische Analysen und Darstellungen gefragt. Diese hätten zeigen können, dass Ländlichkeit eben auch praktisch anders geht. Das Europäische Ländliche Parlament und die europäische Dorfbewegung, die sich mit dem Slogan „Recht auf Dorf“ und dem europäischen ländlichen Manifest seit mehr als 15 Jahren für die Demokratisierung ländlicher Verhältnisse engagiert haben, wären hier zu erwarten gewesen, wenngleich sie in der Literatur wenig Beachtung finden. Sind doch Auseinandersetzungen dazu von Halhead und Krambach ebenfalls bei der Rosa-Luxemburg-Stiftung erschienen, wie auch diese Studie in ihrer ersten Veröffentlichung. Auch wenn dieses letzte Kapitel seiner eigenen Utopie nicht gerecht werden kann, ist das gesamte Buch eine beeindruckende Zusammenstellung und Kontextualisierung von Studien zur Komplexität und zu den Herausforderungen des ländlichen Raumes. Formal ermöglicht die gewählte Strukturierung einen klaren Zugang für Neueinsteiger*innen, während theoretische Tiefe und das Aufzeigen von blinden Flecken und Forschungsfeldern auch Alteingesessenen neue Wege weisen. Zusammenfassungen der zentralen Begriffe und Argumente am Ende aller Kapitel machen es für die Lehre besonders ansprechend. Ein Buch, das es aufgrund seiner Vielschichtigkeit und Strukturiertheit verdient, das einführende Standardwerk und Stein des Anstoßes in einer neuen kritischen Betrachtung und Politik ländlicher Räume im deutschsprachigen Kontext zu werden. 